\title{
Effect of Chain Wheel Shape on Crank Torque, Freely Chosen Pedal Rate, and Physiological Responses during Submaximal Cycling
}

\author{
Ernst Albin Hansen ${ }^{1)}$, Kurt Jensen ${ }^{2)}$, Jostein Hallén ${ }^{1)}$, John Rasmussen ${ }^{3)}$ and Preben K. Pedersen ${ }^{4)}$ \\ 1) Department of Physical Performance, The Norwegian School of Sport Sciences, Oslo, Norway \\ 2) Team Danmarks Testcenter, University of Southern Denmark, Odense, Denmark \\ 3) Institute of Mechanical Engineering, Aalborg University, Denmark \\ 4) Institute of Sports Science and Clinical Biomechanics, University of Southern Denmark, Odense, Denmark
}

\begin{abstract}
The development of noncircular chain wheels for the enhancement of cycling performance has been in progress for a long time and continues apace. In this study we tested whether submaximal cycling using a non-circular (Biopace) versus a circular chain wheel resulted in lower peak crank torque at preset pedal rates as well as resulting in lower pedal rate and metabolic response at freely chosen pedal rate. Ten trained cyclists (mean \pm SD: $27 \pm 3$ years of age, $182 \pm 4 \mathrm{~cm}$ tall, $77.5 \pm 7.0 \mathrm{~kg}$ of body mass, and peak oxygen uptake of $61.7 \pm 4.4 \mathrm{ml} \mathrm{kg}^{-1} \mathrm{~min}^{-1}$ ) cycled with a Biopace and a circular chain wheel at $180 \mathrm{~W}$ at 65 and $90 \mathrm{rpm}$ for recording of crank torque profiles, and at their freely chosen pedal rate for recording of pedal rate and metabolic response, including oxygen uptake and blood lactate concentration. Crank torque profiles were similar between the two chain wheels during cycling at preset pedal rates. During cycling at the freely chosen pedal rate (being $93 \pm 6$ and $93 \pm 4 \mathrm{rpm}$ for the Biopace and circular chain wheel, respectively), blood lactate concentration was significantly different between the two chain wheels, being on average $0.2 \mathrm{mmol}^{-1}$ lower with the Biopace chain wheel. A musculoskeletal simulation model supported the idea that a contributing factor to the observed difference in blood lactate concentration may be slightly reduced muscle activity around the phase where peak crank torque occurs during cycling with the Biopace chain wheel. In that particular phase of the crank revolution, the observed slightly lower muscle activity may result from larger transfer of energy from the legs to the crank. J Physiol Anthropol 28(6): 261-267, 2009 http://www.jstage.jst.go.jp/browse/jpa2
\end{abstract}

[DOI: 10.2114/jpa2.28.261]

Keywords: biopace, cycling economy, elliptical chain wheel, noncircular chain ring

\section{Introduction}

More than twenty-five years ago, Okajima (1983) enthusiastically described how his research group at Shimano Corporation aimed to improve the application of the human as bicycle engine by inducing an appropriately uneven crank angular velocity pattern with a non-circular chain wheel. The product of their effort, called the Biopace chain wheel, is best described as a skewed ellipse with nonperpendicular minor and major axes, the latter being close to the crank arms (for a detailed description and depiction, see Cullen et al., 1992, and Hull et al., 1992). Okajima (1983) presented arguments (which will not be repeated here) in favor of the notion that the Biopace chain wheel better optimized the efficiency of the leg muscle work than the circular chain wheel. An increased efficiency would lower the rate of oxygen uptake $\left(\mathrm{VO}_{2}\right)$ at a given low to moderate speed or power output, which is generally considered advantageous for prolonged cycling (Abbiss and Laursen, 2005; Jeukendrup et al., 2000). However, it has been reported that $\mathrm{VO}_{2}$ measured during submaximal cycling at fixed power output and preset pedal rate was not significantly different between the Biopace and circular chain wheels (Cullen et al., 1992). Thus, the shape of the Biopace chain wheel per se does not appear to improve muscular efficiency.

It was also stated by Okajima (1983) that peak joint torques of the legs, as calculated from pedal forces, would be reduced by using a Biopace instead of a traditional circular chain wheel. Unfortunately, data from only one subject were presented. Therefore, the results reported by Okajima (1983) should be taken with some reservation and more comprehensive data on crank torque profile and pedal force are required to make such a conclusion. One study (Neptune and Herzog, 2000) supports the hypothesis of a lower peak crank torque $\left(\mathrm{T}_{\text {peak }}\right)$ during cycling with the Biopace versus a circular chain wheel. That study (Neptune and Herzog, 2000) showed lower $\mathrm{T}_{\text {peak }}$ during cycling with an elliptical chain wheel than 
with a circular chain wheel. Though not identical to the Biopace, the employed chain wheel in the study by Neptune and Herzog (2000) possessed some of the same characteristics, with its major axis being oriented along the crank arms.

The magnitude of $\mathrm{T}_{\text {peak }}$ may be of importance for an individual's choice of pedal rate during cycling since peak tension development may dominate effort sensation (Mihevic, 1981). A reduction of $T_{\text {peak }}$, which occurs, for example, when the crank inertial load is reduced during cycling at a fixed power output (Fregly et al., 1996; Hansen et al., 2002), has therefore been proposed to explain why cyclists choose a slightly lower pedal rate at low compared to high crank inertial load (Hansen et al., 2002). This could potentially reduce the oxygen demand since the freely chosen pedal rate during submaximal cycling is generally higher than the one that results in minimum $\mathrm{VO}_{2}$ (Nielsen et al., 2004). Hence, if $\mathrm{T}_{\text {peak }}$ is indeed reduced with the Biopace chain wheel as implied by Okajima (1983), it is possible that both freely chosen pedal rate and, consequently, internal power (performed on particularly the legs, to move these through each crank revolution) and metabolic response including $\mathrm{VO}_{2}$ (Hansen et al., 2004; Tokui and Hirakoba, 2008), would be lower during cycling with a Biopace chain wheel than with a circular chain wheel. This could reduce the energetic load and perhaps even improve endurance performance during prolonged submaximal cycling. Freely chosen pedal rate has not previously been compared between Biopace and circular chain wheels of the same size. In light of this and the above reasoning it appears therefore that "the Biopace case" may deserve to be reopened. We therefore implemented the present study. The purpose was to test the hypotheses that 1) $T_{\text {peak }}$ at preset pedal rates of 65 and $90 \mathrm{rpm}$ would be lower when using a non-circular (Biopace) as opposed to a circular chain wheel, and 2) that the freely chosen pedal rate would be lower with the non-circular chain wheel, which secondarily might reduce $\mathrm{VO}_{2}$.

\section{Methods}

\section{Subjects}

Ten trained competitive cyclists $(27 \pm 3$ years, $182 \pm 4 \mathrm{~cm}$, and $77.5 \pm 7.0 \mathrm{~kg}$ ) participated in the study, which was approved by the local ethics committee. The subjects signed an informed consent form prior to participation. To minimize the subjects' focus on their choice of pedal rate, they were not informed of the purpose of the study, but were told that the test session they participated in was merely a pre-test for some additional experiments that they had volunteered for. After the subjects had completed their tests, they were debriefed about the actual purpose as well as the results of the study.

\section{Procedures}

After a 30-min warm up and a 5-min pause, the subjects performed a 10 -min bout at $180 \mathrm{~W}$ with either a 52 -tooth noncircular Biopace II (CR-BP20, Shimano, Osaka, Japan) or a 52-tooth circular chain wheel, in both cases at a freely chosen pedal rate. The reason for choosing such a submaximal intensity was that $70 \%$ of long ( $>5 \mathrm{~h}$ ) competitive road races consists of submaximal cycling below approximately $60-70 \%$ of peak $\mathrm{VO}_{2}$ (Broker, 2003; Lucia et al., 1999). Thus, despite the submaximal character of this work, it represents a substantial part of the race and is therefore assumed to contribute considerably to the development of fatigue. Then, after a 5-min pause, another 10-min bout with the alternate chain wheel was performed. The order of the chain wheels was counterbalanced to avoid an order effect. During the final $5 \mathrm{~min}$ of these 10-min bouts, the means of the following variables were calculated: freely chosen pedal rate, $\mathrm{VO}_{2}$, respiratory exchange ratio, and heart rate. During the final minute, blood lactate concentration ([La]) was measured and the rating of perceived exertion was recorded.

After another 5-min pause, the subjects performed two 2 -min bouts at $180 \mathrm{~W}$ at target pedal rates of 65 and $90 \mathrm{rpm}$ with either the Biopace or the circular chain wheel for determination of crank torque profile characteristics. Immediately after the second 2-min bout, two identical bouts were performed with the alternate chain wheel. The order of the chain wheels was counterbalanced.

Finally, in order to obtain a measure of the subjects' general fitness level they performed after a 5-min pause a single 5-min all-out test at freely chosen pedal rate for determination of the average power output $\left(\mathrm{W}_{5 \min }\right)$, pedal rate, as well as peak values of $\mathrm{VO}_{2}$, respiratory exchange ratio, and heart rate. Peak values of $\mathrm{VO}_{2}$ and respiratory exchange ratio represent the highest means of $30 \mathrm{~s}$ sample periods. [La] was measured 1 and 3 min after termination of this all-out test and the highest value was considered to represent peak. This all-out test was performed with a circular chain wheel.

\section{Instrumentation}

Cycling was performed on an electromagnetic SRM ergometer that was mounted with a Science version of the crank dynamometer (Schoberer Rad Messtechnik, Julich, Germany). The cycle ergometer was adjusted to conform to the measurements of the cyclists' own racing bicycles and the cyclists used their own cycling shoes and pedals. The SRM ergometer sampled power output and pedal rate at $1 \mathrm{~Hz}$ and also allowed measurement of crank torque (sum of the torque applied by left and right leg). For determination of crank torque profile characteristics, crank torque was recorded twice at each 2-min cycling condition, at $500 \mathrm{~Hz}$ for $15 \mathrm{~s}$. For each of the two recordings, an average crank torque profile for one crank cycle was calculated by the SRM software. These crank torque profiles were analysed for the three crank torque characteristics of $\mathrm{T}_{\text {peak }}, \mathrm{T}_{\text {min }}$, as well as crank angle at $\mathrm{T}_{\text {peak }}$ (Hansen et al., 2002). Then, for each subject, cycling condition, and crank torque characteristic, a mean of the two obtained values was calculated, for further analysis. During the submaximal cycling, "gear 9" and the "constant Watt" operating mode of the SRM ergometer were used. When operating in this mode, the ergometer maintains a constant 
power output, regardless of pedal rate. For the 5-min all-out test, the "open end test" operating mode was used with the subjects starting in "gear 9" and being allowed to change gear. In this mode, the power output is changed if either pedal rate is changed or the gear is changed at a constant pedal rate.

$\mathrm{VO}_{2}$ and respiratory exchange ratio were determined with an AMIS 2001 metabolic cart (Innovision, Odense, Denmark). Gas analysers were calibrated against certified gases of known concentrations, and ventilation sensors were calibrated with a 3-liter syringe. Heart rate was measured with a Polar S610 heart rate monitor (Electro Oy, Kempele, Finland). [La] was measured in fingertip blood samples using a YSI Model 1500 Sport, with a precision of $\pm 2 \%$ of reading or $0.1 \mathrm{mmol}^{-1}$, whichever is larger (YSI, Inc., Yellow Springs, Ohio, USA). Rating of perceived exertion was indicated on Borg's 6-20 scale (Borg, 1970).

\section{Statistics}

Paired t-tests were performed to evaluate differences in measured variables between the Biopace and circular chain wheel at freely chosen pedal rate (StatView 5.0, SAS Institute, Inc., NC, USA). Repeated measures ANOVA with chain wheel and pedal rate as factors, followed by paired t-tests (post hoc), were used to evaluate differences in the measured variables from the part of the study with preset pedal rate. A Pearson product-moment correlation coefficient test was used to analyze the relationship between [La] during cycling with the circular chain wheel and decrease in [La] with the Biopace chain wheel (Excel 2003, Microsoft Corp., WA, USA). The significance level was set at $p \leq 0.05$. Data are presented as mean \pm SD unless otherwise indicated.

\section{Results}

The measured $\mathrm{T}_{\text {peak }}$ and $\mathrm{T}_{\text {min }}$ values during cycling at preset pedal rates were significantly lower at $90 \mathrm{rpm}$ than at $65 \mathrm{rpm}$ $\left(p<0.05\right.$; Table 1). In addition, $\mathrm{T}_{\text {peak }}$ occurred later in the crank revolution at $90 \mathrm{rpm}$ compared with $65 \mathrm{rpm}(p<0.05)$. But there was no significant effect of chain wheel shape on any of these measured variables.

The pedal rates during the submaximal cycling trials at freely chosen pedal rate were not significantly different between the two chain wheels, nor were there any significant differences in $\mathrm{VO}_{2}$, respiratory exchange ratio, heart rate, and rating of perceived exertion (Table 2). [La], on the other hand, was significantly lower during cycling with the Biopace chain wheel, although the difference in absolute values was small (on average $0.2 \mathrm{mmol}^{-1}$ ) (Fig. 1, Table 2). The subjects who had the highest [La] during cycling with the circular chain wheel showed the largest decrease in [La] with the Biopace chain wheel $(\mathrm{r}=0.86, p<0.05)$.

The 5-min all-out test confirmed that the subjects were well trained (Peak $\mathrm{VO}_{2}>60 \mathrm{ml} \mathrm{kg}^{-1} \mathrm{~min}^{-1}$, and $\mathrm{W}_{5 \min }>400 \mathrm{~W}$, Table 3). Power output during the submaximal cycling at freely

Table 1 Crank torque profile characteristics obtained during submaximal cycling $(180 \mathrm{~W})$ at preset pedal rates with circular and Biopace chain wheel $($ mean \pm SD)

\begin{tabular}{lccc}
\hline & & \multicolumn{2}{c}{ Target pedal rate (rpm) } \\
\cline { 3 - 4 } & & 65 & 90 \\
\hline \multirow{2}{*}{ Pedal rate $(\mathrm{rpm})$} & Circular & $64.6 \pm 1.1$ & $89.9 \pm 1.5^{*}$ \\
& Biopace & $64.7 \pm 1.6$ & $88.9 \pm 1.7^{*}$ \\
$\mathrm{~T}_{\text {peak }}(\mathrm{Nm})$ & Circular & $40.4 \pm 3.9$ & $29.0 \pm 2.6^{*}$ \\
$\mathrm{~T}_{\text {min }}(\mathrm{Nm})$ & Biopace & $41.1 \pm 4.0$ & $29.3 \pm 2.0^{*}$ \\
\multirow{2}{*}{ Crank angle at $\mathrm{T}_{\text {peak }}($ degrees $)$} & Circular & $6.0 \pm 1.4$ & $4.5 \pm 1.3^{*}$ \\
& Biopace & $5.7 \pm 1.5$ & $4.7 \pm 0.9^{*}$ \\
& Circular & $79 \pm 7$ & $89 \pm 8^{*}$ \\
& Biopace & $80 \pm 8$ & $90 \pm 9^{*}$ \\
\hline
\end{tabular}

*Different from $65 \mathrm{rpm}(p<0.05)$

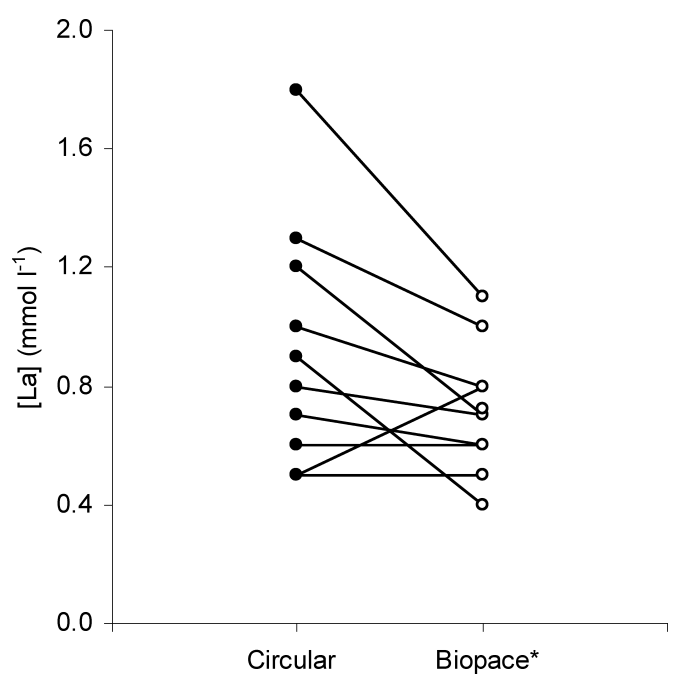

Fig. 1 Blood lactate concentration ([La]) obtained during submaximal cycling at $180 \mathrm{~W}$ with circular and Biopace chain wheel. Individual data. $*$ Different from circular $(p<0.05)$.

Table 2 Biomechanical and physiological responses during submaximal cycling at $180 \mathrm{~W}$ at freely chosen pedal rate with circular and Biopace chain wheel (mean $\pm \mathrm{SD})$

\begin{tabular}{|c|c|c|c|c|c|c|}
\hline & $\begin{array}{c}\text { Freely chosen } \\
\text { pedal rate } \\
(\mathrm{rpm})\end{array}$ & $\begin{array}{c}\mathrm{VO}_{2} \\
\left(1 \mathrm{~min}^{-1}\right)\end{array}$ & $\begin{array}{c}\text { Respiratory } \\
\text { exchange ratio }\end{array}$ & $\begin{array}{c}\text { Heart rate } \\
\left(\text { beats } \min ^{-1} \text { ) }\right.\end{array}$ & $\begin{array}{c}{[\mathrm{La}]} \\
\left(\mathrm{mmol}^{-1}\right)\end{array}$ & $\begin{array}{l}\text { Rating of perceived } \\
\text { exertion }\end{array}$ \\
\hline Biopace & $93 \pm 6$ & $2.13 \pm 0.09$ & $0.91 \pm 0.06$ & $118 \pm 11$ & $0.7 \pm 0.2 *$ & $10.3 \pm 1.6$ \\
\hline
\end{tabular}

*Different from the circular chain wheel $(p<0.05)$. 
Table 3 Results from the 5-min all-out test (mean \pm SD)

\begin{tabular}{ccccc}
\hline $\begin{array}{c}\mathrm{W}_{5 \min } \\
(\mathrm{W})\end{array}$ & $\begin{array}{c}\text { Peak } \mathrm{VO}_{2} \\
\left(\mathrm{ml}^{-1} \mathrm{~kg}^{-1} \mathrm{~min}^{-1}\right)\end{array}$ & $\begin{array}{c}\text { Peak respiratory } \\
\text { exchange ratio }\end{array}$ & $\begin{array}{c}\text { Peak heart rate } \\
\left(\text { beats } \mathrm{min}^{-1}\right)\end{array}$ & $\begin{array}{c}\text { Peak [La] } \\
\left(\mathrm{mmoll}^{-1}\right)\end{array}$ \\
\hline $411 \pm 28$ & $61.7 \pm 4.4$ & $1.19 \pm 0.13$ & $186 \pm 9$ & $13.1 \pm 2.7$ \\
\hline
\end{tabular}

chosen pedal rate corresponded to $43 \pm 3 \%$ of the $\mathrm{W}_{5 \text { min }}$ hereas $\mathrm{VO}_{2}$ corresponded to $45 \pm 4 \%$ of the peak $\mathrm{VO}_{2}$, and heart rate corresponded to $63 \pm 4 \%$ of the peak heart rate.

\section{Discussion}

Crank torque profile characteristics were different during submaximal cycling between $65 \mathrm{rpm}$ and $90 \mathrm{rpm}$, yet there was no significant difference between the two chain wheels in either case. Thus, the results reject our first hypothesis that $\mathrm{T}_{\text {peak }}$ would be lower during cycling at preset pedal rates with the Biopace compared with a conventional circular chain wheel. This hypothesis was motivated by Okajima's (1983) description of lower peak joint torques in the legs (calculated from pedal forces) during cycling with the Biopace chain wheel. The hypothesis was further motivated by a study (Neptune and Herzog, 2000) showing lower $\mathrm{T}_{\text {peak }}$ during cycling with an elliptical versus a circular chain wheel, although the ratio between the major and minor axes of the elliptical chain wheel used by Neptune and Herzog (2000) was considerably larger (1.36) than for a 52-tooth Biopace chain wheel (1.04), possibly resulting in an increasing alteration of the crank torque profile. Of note is the fact that the ratio between the lengths of the major and minor axes of the Biopace chain wheel increases with decreasing tooth number, reaching a maximum of 1.17 for a 28-tooth chain wheel (Cullen et al., 1992; Okajima, 1983). Okajima (1983) did not report the tooth number of the chain wheels that were used for collection of the joint torque data he presented. However, based on his finding of a difference in joint torques between the Biopace and circular chain wheel, it seems likely that the ratio between the major and minor axes was larger (i.e., the chain wheel was smaller) than in the present study. If this is the case, then the tooth number, and thereby the ratio between the major and minor axes of a non-circular chain wheel, likely influences the magnitude of the differences in peak pedal force, peak crank torque, and peak joint torques in the legs that may be found when comparing such a chain wheel shape with a circular chain wheel. Furthermore, the present application of relatively large chain wheels (52-tooth) could be the reason why no differences in crank torque profile characteristics were found. However, the 52-tooth chain wheels were chosen for the present study since this chain wheel size is required during fast cycling $(>35 \mathrm{~km} / \mathrm{h})$, which makes it mandatory for road races. For examples of crank torque profiles, the reader is referred to a previously published paper from our group (Hansen et al., 2002).

The second hypothesis, that the subjects would prefer a lower pedal rate when cycling with the Biopace chain wheel, was based on the expectation that $\mathrm{T}_{\text {peak }}$ would be lower with the Biopace chain wheel. Since the latter turned out not to be the case, it seems logical that the freely chosen pedal rate did not differ between the chain wheels. Oxygen consumption was also similar, as was heart rate and rating of perceived exertion. Others have also reported similar aerobic loads during cycling with Biopace and circular chain wheels (Cullen et al., 1992). In the study by Cullen et al. (1992), cyclists exercised with 42and 52-tooth Biopace and circular chain wheels at preset pedal rates of 50,70 , and $90 \mathrm{rpm}$ at work loads resulting in intensities between $29 \%$ and $83 \%$ of peak $\mathrm{VO}_{2}$. Heart rate, $\mathrm{VO}_{2}$, and rating of perceived exertion were not different between the chain wheels in that study. Hull et al. (1992) reported that cyclists chose on average 5-6 rpm higher pedal rates during cycling at $60-80 \%$ of peak $\mathrm{VO}_{2}$ with a Biopace than with a circular chain wheel. However, in that study the two chain wheels were not directly comparable since a 42tooth Biopace and a 52-tooth circular chain wheel were used. Even though the gear ratio was kept similar between the two chain wheel shapes, the effect of chain wheel size on the crank torque profile, perceived exertion, and choice of pedal rate is unknown.

A surprising finding of the present study was that [La] was significantly lower during cycling with the Biopace chain wheel than with the circular chain wheel. Of note is the fact that the subjects were allowed to choose pedal rate during cycling and did in fact, on average, employ similar pedal rates with the two chain wheels. Thus, the difference in lactate response cannot be attributed to a difference in pedal rate, which otherwise has been reported to influence lactate response during cycling (Böning et al., 1984). Even when some subjects chose pedal rates that were up to $7 \mathrm{rpm}$ different between the two chain wheels, a lower [La] with the Biopace chain wheel occurred systematically. Additionally, as all subjects trained and competed with a circular chain wheel and were not accustomed to the Biopace chain wheel, the [La] result should not be attributed to habituation. In the case of habituation, one would rather expect a lower [La] during cycling with the familiar circular chain wheel. One possibility is that cycling with the Biopace chain wheel requires less muscle activity, affecting muscle fibre recruitment and reducing lactate production. The present finding that the subjects who had the highest [La] during cycling with the circular chain wheel showed the largest decrease in [La] with the Biopace chain wheel might be interpreted in support of this. It is reasonable to suppose that, given the fact that the Biopace chain wheel reduces [La], those subjects who had a low [La] close to a minimal value, only had the potential to reduce their [La] marginally.

In the generation of the pedal force or crank torque, the force produced by the muscles is superimposed on nonmuscular contributions from the inertial forces of the legs, which may diverge between the two chain wheels. It is therefore possible that, even when the measured crank torque 
profile characteristics are similar for the Biopace and circular chain wheels, as here, the ratio between the muscular and nonmuscular contributions to $\mathrm{T}_{\text {peak }}$, and the crank torque in general, differs between the two chain wheels. The reason is that during cycling with the Biopace chain wheel, a larger acceleration of the crank and the pushing leg occurs in the first quarter of the crank revolution due to the decreasing chain wheel radius in this phase (Hull et al., 1992) (see also Fig. 2). If the larger amount of stored kinetic energy in the leg from this acceleration is subsequently transferred to the crank, around the crank angle where $\mathrm{T}_{\text {peak }}$ occurs, it may reduce the required muscle activity in this phase. In Appendix A, the plausibility of this hypothesis is investigated by means of a musculoskeletal simulation model that integrates theoretical and experimental work to improve the evaluation of the suggested physiological explanations of the observed results. The results of the computer simulation may support the hypothesis, although the simulated difference between the two chain wheels is marginal, as could be expected from the small obliquity of the 52-tooth Biopace wheel. A reduced muscle activity level with the Biopace chain wheel around the phase where $T_{\text {peak }}$ occurs may be associated with less fast twitch muscle fibre activity, which could explain the lower [La]. The background for this suggestion is the general acceptance of the level of required muscle tension to determine the extent of slow and fast twitch muscle fibre recruitment in an orderly fashion (Henneman et al., 1965; Henneman and Olson, 1965), such that slow twitch fibres are predominantly recruited in aerobic-type low-force activities, and fast twitch fibres are gradually recruited as activities increase toward anaerobic-type sprint activities and other forceful muscle actions. The fast twitch muscle fibres, compared with the slow twitch fibres, are characterized by their larger dependency on glycolytic degradation of glycogen to lactate for ATP production (i.e., anaerobic metabolism). Thus, the muscle fibres' tendency to produce lactate increases in the same order as the recruitment pattern (i.e., slow twitch $<$ fast twitch) (Gladden, 1996).

Although the effect of chain wheel shape on [La] was large in terms of percentage change in the present study, the absolute magnitude of the change was modest. Therefore, one should be cautious when speculating on the physiological significance of using one chain wheel instead of the other. Nevertheless, if it is assumed that the lower [La] with the Biopace chain wheel represents a lower anaerobic load, it may be considered that depletion of the energy resources that are available in limited amounts, such as muscle and liver glycogen, occurs at a slower rate with the Biopace chain wheel, delaying the onset of fatigue (Sahlin et al., 1998). Another consideration is that if fast twitch muscle fibre recruitment is reduced with the Biopace chain wheel, sprint capability at the end of prolonged cycling could be better preserved. This would be advantageous since the outcome of a cycling road race is frequently determined in a sprint.

In conclusion, the two initial hypotheses were rejected. That is, the Biopace chain wheel neither reduced $\mathrm{T}_{\text {peak }}$ (at preset pedal rates), nor did it cause a reduced freely chosen pedal rate and $\mathrm{VO}_{2}$. However, [La] was lower during submaximal cycling with the Biopace chain wheel. The simulation model showed that the altered chain wheel shape, all other things being equal, may slightly change the muscle activity pattern and this might contribute to explain the experimental findings (of lower [La]). It could be speculated that the altered activity pattern slightly reduces the recruitment of fast twitch muscle fibres around the phase where $T_{\text {peak }}$ occurs during cycling with the Biopace chain wheel due to deceleration of the leg segment masses at this point and the resulting forces from the feet on the pedals. However, the effect is minor and it remains unclear whether it is significant compared to factors not considered in the simulation model.

\section{Appendix A}

This appendix is an attempt to quantify by means of a computational model the contribution from the contracting muscles (as opposed to the non-muscular contribution) to the crank torque around the phase where $\mathrm{T}_{\text {peak }}$ occurs. The use of a computational model is motivated by the fact that it is noisefree and therefore allows assessment of model tendencies that are too small to be reliably picked up in an experiment.

A musculoskeletal model of the legs and pelvis attached to a crank mechanism and a seat is developed in version 2.0 of the AnyBody Modeling System (AnyBody Technology A/S, Aalborg, Denmark). Each leg comprises a rigid foot, a shank, and a thigh. The ankle joint is a universal joint, the knee joint is a hinge joint, and the hip joint is a spherical joint. The model is equipped with a total of 92 individually activated muscle units to execute an imposed crank torque profile that was obtained from a subject in the present study at $180 \mathrm{~W}$ and $90.8 \mathrm{rpm}$ and approximated by a least squares fit to a double sine function. Muscle configuration and individual muscle unit parameters largely follow previous work (Delp, 1995) with the addition of several branches of $\mathrm{m}$. psoas major (Hansen et al., 2006). Furthermore, each muscle unit comprises a contractile element, a parallel-elastic element, and a serial-elastic element, and as such takes passive elasticity in the tissues and contraction dynamics into account. The muscle recruitment is based on inverse dynamics using a $\mathrm{min} / \mathrm{max}$, i.e., a minimum fatigue, recruitment criterion (An et al., 1984; Bean et al., 1988; Rasmussen et al., 2001). Minimum fatigue has previously shown good agreement with experimental data for muscle coordination in bicycling (Prilutsky and Gregor, 2000).

In addition to the imposed crank torque, the model takes into account the inertia forces and gravity forces affecting the legs. The computational model presumes the same crank torque profile for the two chain wheels since no measurable difference between the two chain wheels was found experimentally. Furthermore, the model presumes a constant bicycle velocity leading to a constant crank angular velocity in the case of the circular chain wheel.

The Biopace chain wheel was scanned using a flatbed 


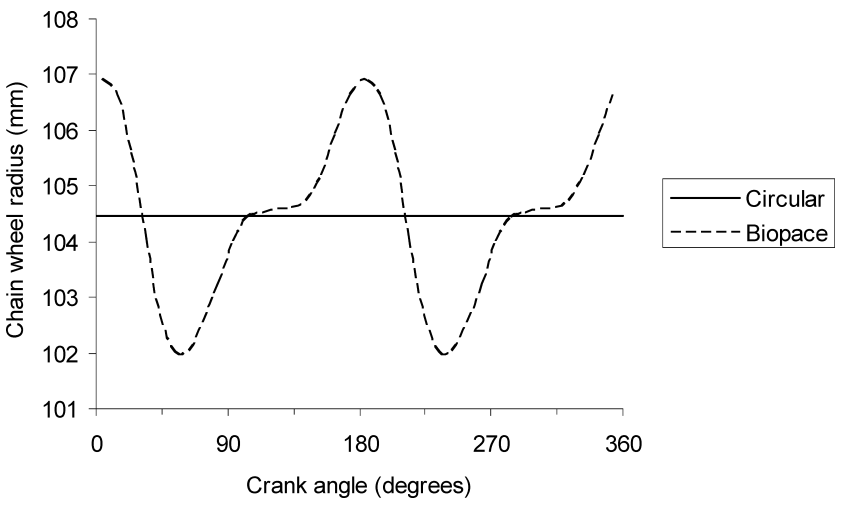

Fig. 2 Chain wheel radius measured from the root of each tooth to the centre of the chain wheel as a function of crank angle for the circular and Biopace chain wheel.

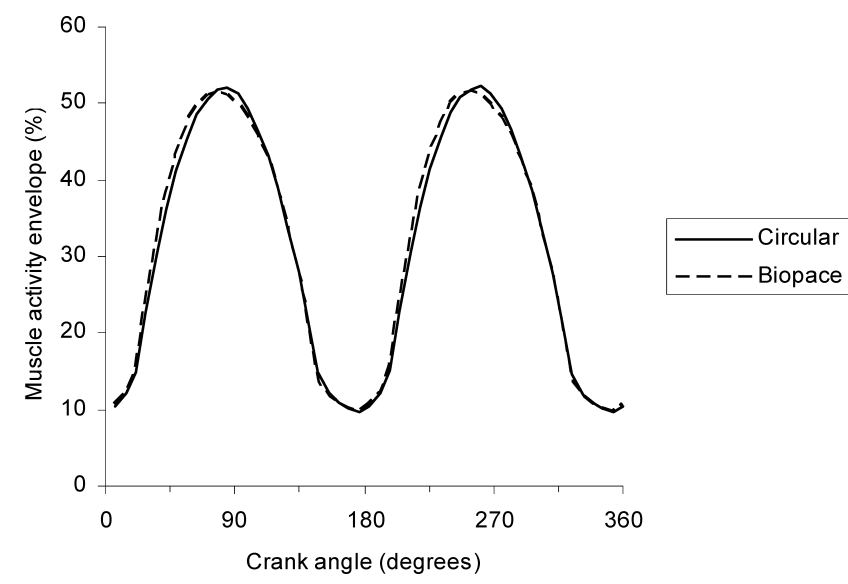

Fig. 3 Muscle activity envelope as a function of crank angle for the circular and Biopace chain wheel.

scanner at a resolution of $1650 \times 1740$ pixels. The image was imported into a computer-aided design system and scaled to the physical size of the original chain wheel. A spline curve was then fitted to the root of each tooth on the chain wheel and radii for each 10 degrees were digitized (Fig. 2). By means of the digitized radii and the assumption of constant bicycle and chain velocity, the crank velocity variation for the Biopace chain wheel was computed. The constant crank velocity for the circular chain wheel as well as the variable speed for the Biopace chain wheel were then imposed in turn on the model to simulate the muscle activity

The nature of the $\min / \max$ criterion (Rasmussen et al., 2001) is to cause maximum synergism between muscle units, taking into account each muscle unit's maximal contraction force. More precisely, this leads to the concept of muscle activity as the ratio between developed force in the muscle's contractile elements and the muscle's maximal contraction force, taking muscle length and contraction velocity into account.

This is mathematically equivalent to a minimization of the maximum muscle activity at each time step of the simulation and consequently tends to share the maximum activity at any time in the simulation between several synergistic muscle units, creating a clearly defined curve enveloping the activities of all muscle units in the model. This muscle activity envelope therefore reflects how strenuous the task is for the system of involved muscle units as a whole at any point in the simulation.

A comparison of the muscle activity envelope as a function of the crank angle during cycling with the two chain wheels shows that the Biopace chain wheel allows development of the specified crank torque at a slightly reduced muscle activity at the points of $\mathrm{T}_{\text {peak }}$ provided the central nervous system recruits muscles at maximum synergism (Fig. 3). Further, it appears that the rate of increase of muscle activity (slope of curve) just before the crank angle where $T_{\text {peak }}$ occurs is slightly smaller with the Biopace chain wheel. Both aspects may support the present suggestion that the muscular part of the contribution to the crank torque just before and at $\mathrm{T}_{\text {peak }}$ could be slightly smaller with the Biopace chain wheel than with the circular chain wheel, although the differences are marginal, as could be expected from the weak obliquity of the Biopace chain wheel.

The major assumption of the current simulations is that the Biopace chain wheel does not impose any change of kinematics in the cyclist except for a slight variation in crank velocity over a cycle. The advantage of the model under these assumptions is that it does not have other sources of noise and can therefore reveal very small differences, as those depicted in Fig. 3. Whether these are the actual cause of the observed experimental differences in [La] cannot be verified by the model.

\section{References}

Abbiss CR, Laursen PB (2005) Models to explain fatigue during prolonged endurance cycling. Sports Med 35: 865898

An KN, Kwak BM, Chao EY, Morrey BF (1984) Determination of muscle and joint forces: A new technique to solve the indeterminate problem. Trans ASME 106: 364 367

Bean JC, Chaffin DB, Schultz AB (1988) Biomechanical model calculation of muscle contraction forces: a double linear programming method. J Biomech 21: 59-66

Böning D, Gönen Y, Maassen N (1984) Relationship between work load, pedal frequency, and physical fitness. Int J Sports Med 5: 92-97

Borg G (1970) Perceived exertion as an indicator of somatic stress. Scand J Rehab Med 2: 92-98

Broker JP (2003) Cycling power: road and mountain. In Burke ER ed. High-tech cycling. Human Kinetics, 147-174

Cullen LK, Andrew K, Lair KR, Widger MJ, Timson BF (1992) Efficiency of trained cyclists using circular and noncircular chainrings. Int J Sports Med 13: 264-269

Delp SL (1995) http://www.isbweb.org/data/delp/

Fregly BJ, Zajac FE, Dairaghi CA (1996) Crank inertial load has little effect on steady-state pedaling coordination. J 
Biomech 29: 1559-1567

Gladden LB (1996) Lactate transport and exchange during exercise. In Rowell L, Shephard JT eds. Handbook of physiology. Exercise: Regulation and integration of multiple systems. Am Physiol Soc, New York, 614-648

Hansen EA, Jørgensen LV, Jensen K, Fregly BJ, Sjøgaard G (2002) Crank inertial load affects freely chosen pedal rate during cycling. $\mathrm{J}$ Biomech 35: 277-285 [Erratum in J Biomech 35: 1521 (2002)]

Hansen EA, Joørgensen LV, Sjøgaard G (2004) A physiological counterpoint to mechanistic estimates of "internal power" during cycling at different pedal rates. Eur J Appl Physiol 91: 435-442

Hansen L, de Zee M, Rasmussen J, Andersen TB, Wong C, Simonsen EB (2006) Anatomy and biomechanics of the back muscles in the lumbar spine with reference to biomechanical modeling. Spine 31: 1888-1899

Henneman E, Olson CB (1965) Relations between structure and function in the design of skeletal muscles. J Neurophysiol 28: 581-598

Henneman E, Somjen G, Carpenter DO (1965) Functional significance of cell size in spinal motoneurons. J Neurophysiol 28: 560-580

Hull ML, Williams M, Williams K, Kautz S (1992) Physiological response to cycling with both circular and noncircular chainrings. Med Sci Sports Exerc 24: $1114-1122$

Jeukendrup AE, Craig NP, Hawley JA (2000) The bioenergetics of World Class Cycling. J Sci Med Sport 3: 414-433

Lucia A, Hoyos J, Carvajal A, Chicharro JL (1999) Heart rate response to professional road cycling: The Tour de France.
Int J Sports Med 20: 167-172

Mihevic PM (1981) Sensory cues for perceived exertion: a review. Med Sci Sports Exerc 13: 150-163

Neptune RR, Herzog W (2000) Adaptation of muscle coordination to altered task mechanics during steady-state cycling. J Biomech 33: 165-172

Nielsen JS, Hansen EA, Sjøgaard G (2004) Pedalling rate affects endurance performance during high-intensity cycling. Eur J Appl Physiol 92: 114-120

Okajima S (1983) Designing chainwheels to optimize the human engine. Bike Tech 2: 1-7

Prilutsky BI, Gregor RJ (2000) Analysis of muscle coordination strategies in cycling. IEEE Transactions on rehabilitation engineering 8: 362-370

Rasmussen J, Damsgaard M, Voigt M (2001) Muscle recruitment by the $\mathrm{min} / \mathrm{max}$ criterion - a comparative numerical study. J Biomech 34: 409-415

Sahlin K, Tonkonogi M, Söderlund K (1998) Energy supply and muscle fatigue in humans. Acta Physiol Scand 162: 261-266

Tokui M, Hirakoba K (2008) Estimation of oxygen cost of internal power during cycling exercise with changing pedal rate. J Physiol Anthropol 27: 133-138

Received: January 30, 2009

Accepted: August 27, 2009

Correspondence to: Ernst Albin Hansen, The Norwegian School of Sport Sciences, Postbox 4014 Ullevål Stadion, NO0806 Oslo, Norway

Phone: +47-23-26-23-30

Fax: +47-22-23-42-20

e-mail: Ernst.albin.hansen@nih.no 